\title{
Gait bradykinesia in Parkinson's disease: a change in the motor program which controls the synergy of gait
}

\author{
Tateo Warabi ${ }^{1} \cdot$ Hiroyasu Furuyama $^{1} \cdot$ Eri Sugai $^{1} \cdot$ Masamichi Kato $^{1} \cdot$ \\ Nobuo Yanagisawa ${ }^{2}$
}

Received: 16 June 2017 / Accepted: 10 October 2017 / Published online: 27 October 2017

(C) The Author(s) 2017. This article is an open access publication

\begin{abstract}
This study examined how gait bradykinesia is changed by the motor programming in Parkinson's disease. Thirty-five idiopathic Parkinson's disease patients and nine age-matched healthy subjects participated in this study. After the patients fixated on a visual-fixation target (conditioningstimulus), the voluntary-gait was triggered by a visual onstimulus. While the subject walked on a level floor, soleus, tibialis anterior EMG latencies, and the $y$-axis-vector of the sole-floor reaction force were examined. Three paradigms were used to distinguish between the off-/on-latencies. The gap-task: the visual-fixation target was turned off; $200 \mathrm{~ms}$ before the on-stimulus was engaged (resulting in a $200 \mathrm{~ms}-$ gap). EMG latency was not influenced by the visual-fixation target. The overlap-task: the on-stimulus was turned on during the visual-fixation target presentation (200 ms-overlap). The no-gap-task: the fixation target was turned off and the on-stimulus was turned on simultaneously. The onset of EMG pause following the tonic soleus EMG was defined as the off-latency of posture (termination). The onset of the tibialis anterior EMG burst was defined as the on-latency of gait (initiation). In the gap-task, the on-latency was unchanged in all of the subjects. In Parkinson's disease, the visual-fixation target prolonged both the off-/on-latencies in the overlaptask. In all tasks, the off-latency was prolonged and the off-/ on-latencies were unsynchronized, which changed the synergic movement to a slow, short-step-gait. The synergy of gait
\end{abstract}

Tateo Warabi

twarabi@mue.biglobe.ne.jp

1 Clinical Brain Research Laboratory, Department of Neurology, Sapporo Yamanoue Hospital, Toyokura Memorial Hall, Yamanote 6-9-1-1, Nishi-ku, Sapporo, Hokkaido 063-0006, Japan

2 Department of Neurology, School of Medicine, Shinshu University, Matsumoto, Japan was regulated by two independent sensory-motor programs of the off- and on-latency levels. In Parkinson's disease, the delayed gait initiation was due to the difficulty in terminating the sensory-motor program which controls the subject's fixation. The dynamic gait bradykinesia was involved in the difficulty (long off-latency) in terminating the motor program of the prior posture/movement.

Keywords Parkinson's disease $\cdot$ Synergy of gait · Latency of electromyogram · Gait dynamics · Sensorymotor programming

$\begin{array}{ll}\text { Abbreviations } \\ \text { LED } & \text { Light-emitting diodes } \\ \text { EMG } & \text { Electromyogram } \\ \text { H-Y } & \text { Hoehn and Yahr } \\ \text { GPi } & \text { Globus pallidus pars interna } \\ \text { GPe } & \text { Globus pallidus pars externa } \\ \text { SNr } & \text { Substantia nigra pars reticulata } \\ \text { STN } & \text { Subthalamic nucleus } \\ \text { DAT } & \text { Dopamine transporters } \\ \text { G } & \text { Gap-task } \\ \text { No-gap } & \text { No-gap-task } \\ \text { Ov } & \text { Overlap-task } \\ \text { off-latency } & \text { Off-EMG latency } \\ \text { on-latency } & \text { On-EMG latency }\end{array}$

\section{Introduction}

The cardinal symptom of Parkinson's disease is difficulty in starting volitional synergic movement; swinging of the arms during walking is diminished, and the gait becomes slow and is usually composed of small steps due to the impaired synergism (Brain and Walton 1969). Movement disorders 
including bradykinesia have been attributed to imbalances in the direct and indirect/hyperdirect pathways in the basal ganglia, resulting in reduced output activity of the cortical motor (Wichman et al. 1994; DeLong and Wichman 2007; Nambu 2008). Since bradykinesia could be due to any one or all of the above factors, the basic question remains. The remained question was the slowness of gait initiation due to difficulty in initiating a new synergic movement or due to difficulty in terminating the preceding posture/movement.

In Parkinson's disease, the slowness of voluntary movement has been ascribed to difficulty with the initiation of movement. Evarts et al. (1981) reported that both reaction time and movement time which both reflect the speed of movement tend to be prolonged in Parkinson's disease. However, the prolongation of reaction time, which reflects the speed of response initiation, is relatively slight. Furthermore, their results are incompatible with the hypothesis that the impairment of the ability to initiate a new movement is the primary cause of bradykinesia. If the timing to terminate a preceding posture/movement is distinguished from the timing to initiate a new volitional movement, it is possible to clarify the difficulty of initiating volitional movements in Parkinson's disease.

Hikosaka and Wurtz (1985) and Hikosaka et al. (1989a, b) conducted the gap and overlap-tasks between two visual signals to separate the neural activity initiating the eye movement from the sensory-motor responses which are related of the neural activity of the primary fixation in the basal ganglia of an awake monkey. In the gap-task, the fixation target was extinguished, and $600 \mathrm{~ms}$ later, the eye movement trigger target was illuminated. In the overlap-task, the subject was required to start the eye movement, while the fixation target was illuminated. Under these conditions, they recorded that the neural activity in the basal ganglia responded to saccade or limb movement, and also responded to visual or auditory stimuli (Hikosaka et al. 1989b). These tasks were useful to separate sensory responses to the target presentation from eye movement-related activity. Warabi et al. (2011) examined a related question in visually guided eye-hand coordinated movements using two types of stimuli (i.e., conditioning and test stimuli) in the gap- and overlaptask conditions which simulated the visual effects of a natural situation. They concluded that the latency prolongation reflected the difficulty in terminating the primary posture arising from the primary cognitive visuospatial situation of the conditioning stimuli. Thus, the start of synergic movement may reflect a combination of involvement between the termination of the preceding posture/movement and the initiation of the new movement.

Numerous studies have addressed instances of gait disturbance in Parkinson's disease subjects and several researchers have examined the initiation of gait (Gantchev et al. 1996; Burleigh-Jacob et al. 1997; Halliday et al. 1998; Carpinella et al. 2007), but ultimately disregarded the effect that the EMG off-response has on the termination of the preceding posture/movement. Most studies focused their analyses on steady-state walking, reporting that leg EMG activity is reduced, and that the results are basically consistent with those observed in arm and finger movements (Evarts et al. 1981; Wing 1988; Cioni et al. 1997; Chee et al. 2009). In addition, it has been reported that the impairment of gait initiation is associated with the visuospatial cognitive environment (Azulay et al. 1999; Cummings and Steven 1992; Naismith et al. 2010).

Carpinella et al. (2007) examined the EMG activity of two antagonist muscles in subjects in the early stage of Parkinson's disease. They reported that the EMG activity in the early stage of Parkinson's disease is basically similar to normal subjects. The steady motor equivalence may operate as the motor program in the motor system of gait. However, the different motor systems do not have a synchronous synergic movement and may emphasize the bradykinesia in the advanced stages of Parkinson's disease (Warabi et al. 1986, 1988). Furthermore, it is unknown how, in the advanced stages of Parkinson's disease, the dynamic gait change is correlated with the unsynchronized motor program between the two antagonist muscles which control termination/initiation.

In the present study, we hypothesized that the dynamic gait initiation changes which are observed in Parkinson's disease are due to the difficulty terminating the preceding motor program. We examined EMG latencies between the soleus and tibialis anterior of the synergic-gait movement which regulate the posture/movement during the dynamic gait initiation in the different stages of Parkinson's disease.

\section{Methods}

\section{Subjects}

Thirty-five patients with idiopathic Parkinson's disease (10 males) and nine age-matched healthy elderly controls (3 males) were examined in this study. The two groups were similar in age (Parkinson's disease: mean $\pm \mathrm{SD}=73.6 \pm 9.6$ years; controls $71.5 \pm 9.5$ years, $p=0.629$ ). Six young control subjects (five males $22.0 \pm 1.9$ years) also participated to test the temporal relationship between sole-floor dynamic reaction-force changes and the EMG activity of the leg muscles (see below). The diagnosis of idiopathic Parkinson's disease was based on the criteria established by the UK Parkinson's Disease Society Brain Bank (Hughes et al. 1992) and the subjects were selected based on similar criteria used in the previous studies (Warabi et al. 1986): specifically, patients whose primary symptom was bradykinesia without hemiparesis, ataxia, or aphasia. The mini-mental state examination scores 
(Folstein et al. 1975) were above the cutoff of 23. Patients who suffered from vascular diseases, degenerative changes, and other neurological diseases combined with essential Parkinson's disease patients were examined and excluded by MRI, SPECT imaging, DAT scan, and comprehensive neurological physical examinations. Patients whose symptoms fluctuated daily in response to L-DOPA treatments were also excluded.

The 35 patients who were selected were divided into three groups based on the severity of their affection by Hoehn-Yahr stage (Hoehn and Yahr 1967) bradykinesia. The classifications were, II moderate $(n=11)$, III severe $(n=13)$, and IV maximum $(n=11)$. Similar to the previous studies (Warabi et al. 1986), all 35 patients were taking levodopa combined with benserazide or carbidopa and dopamine agonists. The dopamine agonists included Pramipexole hydrochloride hydrate, ropinirole hydrochloride, pergolide mesylate, and amantadine hydrochloride. All subjects were fully informed of the purpose and nature of the procedures. Our study complied with the Declaration of Helsinki and was approved by the Sapporo Yamanoue Hospital Ethics Committee.

\section{Experimental design}

All subjects stood and walked on a level floor under normal room lighting while gazing a panel of two vertically arranged light-emitting diodes (LED, each $0.2^{\circ}$ diameter). One was the fixation target (red) which was labeled as the fixation-stimulus (conditioning-stimulus), and the other a two-color LED emitting either a green (go) or a yellow (nogo) stimulus. The two LEDs were located at the top of a $1.2 \mathrm{~m}$-tall pole located at $1.5 \mathrm{~m}$ in front the subject and $30^{\circ}$ to the left of the straight-ahead gaze. We instructed all subjects to maintain an upright posture, placing both heels on the starting line while concentrating on the visual-fixation stimulus. The distance between parallel heels was $30.0 \mathrm{~cm}$; the starting line was $1.5 \times 50.0 \mathrm{~cm}$. After receiving instruction, the subjects stood on the starting line while concentrating on the fixation-stimulus. Of the two test stimuli, the green LED (go-stimulus) instructed the subjects to quickly achieve their own comfortable walking speed (i.e., go), whereas the yellow LED instructed them to remain stationary (i.e., no-go). The yellow LED was randomly included at irregular intervals, and was intended to encourage subjects to pay attention to and respond appropriately to the green test stimulus. In this study, the soleus, tibialis anterior EMG latencies, and the sole-floor reaction force, as measured when walking on a level floor were defined as the parameters of the synergy of gait.

Three paradigms were used to distinguish the difference between the latencies and the gait dynamics of the synergy of gait in each engagement of the fixation and on-stimuli.

\section{Gap}

The fixation-stimulus was turned off $200 \mathrm{~ms}$ before the trigger on-stimulus, and EMG latencies were examined solely based on the trigger on-stimulus without the conditioningstimulus of a fixation target.

\section{Overlap}

During the visual-conditioning-stimulus presentation, the trigger on-stimulus was turned on (200 ms-overlap), and EMG on-latencies, which reflect the effect of the visualconditioning-stimulus, were examined. The overlap-task was examined to examine the effects of the visual cognitive conditioning-stimulus on the EMG latencies.

\section{No-gap}

The fixation-stimulus was tuned off, and simultaneously, the on-stimulus was turned on. All tasks were completed within 25 min, including ten to seven trials of each task. The trials were executed in three sequentially separated blocks.

The $200 \mathrm{~ms}$ delay of the visual-conditioning-stimulus in the overlap-task simulated the effect of a natural situation in which an individual is surrounded and unaware of the visuospatial cognitive environment. This is in contrast to the gap-task which was conducted without a visual-conditioning-stimulus. The subjects usually did not notice the differences between the gap, no-gap, and overlap-tasks (Warabi et al. 2011).

\section{EMG recording and sole-floor reaction force}

EMG activities were recorded from bilateral soleus and tibialis anterior muscles concurrently with sole-floor reactionforces (Kiriyama et al. 2004; Warabi et al. 2004; Warabi and Kato 2009). EMG activity was recorded by bipolar surface electrodes (diameter $15 \mathrm{~mm}$ ) which were placed on each muscle with an inter-electrode distance of $5 \mathrm{~cm}$. Outputs from the EMG electrodes were fed to a multichannel oscillograph (NEC San'Ei, Rectiholy 8K20) through 4-channel EMG amplifiers (Media Area support Co. Type Medi-104 M), and simultaneously stored in a recording unit (TEAC LX-10).

After the gait on-stimulus, a tonic soleus EMG pause represented the termination of the primary posture (Basmajian 1974; Carlsöö 1966). A tonic soleus EMG pause of the noiseless EMG base line was defined as the off-latency (the termination of the primary posture). After the gait onstimulus, the onset of the tibialis anterior EMG burst from the noiseless EMG base line represented the initiation of gait and was defined as the on-latency. The EMG pause between the off- and on-EMG latencies was defined as the off- $I$ 
on-latency difference (interval). The gap- and overlap-task were tested to distinguish the effect of the fixation-stimulus as the conditioning-stimulus. The difference between the EMG latency of the overlap-task and the latency of the gaptask was defined as the conditioning-latency (effect).

We recorded sole-floor dynamic reaction-forces using strain gauge load cells NEC-San'Ei, type 9E01-L42-500 N, diameter $14 \mathrm{~mm}$, and thickness $4 \mathrm{~mm}$ (measuring range 0-500 N), which were secured with double faced adhesive tape on the skin over the medial process of the calcaneus and over the head of the third metatarsal. We used this method to measure the force changes of synergic-gait movement, and compute the $y$-axis vector (see below) during locomotion (Warabi et al. 2004; Warabi and Kato 2009). Since individual sole shape varies remarkably, this method was considered acceptable to evaluate changes in the anatomical $x$-axis and $y$-axis vectors among individual subjects. In this study, only the $y$-axis vectors were analyzed.

For each triggered condition, six trials were recorded. We also recorded trials triggered by an auditory cue (i.e., the gotone). We only examined the gait mode triggered by visual signals in this study. For comparison, we also recorded trials in which subjects initiated gait at their own pace (i.e., selfpaced gait mode) (Brown and Marsden 1988).

\section{Drug therapies in Parkinson's disease}

All 35 Parkinson's disease patients were taking levodopa combined with benserazide or carbidopa and dopamine agonists.

\section{Data analysis}

Output from the gauges was fed to a multichannel pen oscillograph through amplifiers and low-pass filters (frequency range $0-200 \mathrm{~Hz}$ ), and simultaneously stored in the recording unit (TEAC, LX-10). All EMG signals were digitized at $500 \mathrm{~Hz}$.

Subsequent analyses were performed on Windows computers (Microsoft Excel).

\section{Statistical analysis}

We used two-way ANOVA to test the effects of the three triggered conditions (gap, overlap, and no-gap) on four subject groups (elderly controls: $n=9$, Hoehn and Yahr stage II: $n=11$, III: $n=13$, and IV: $n=11$ ). The parameters that were measured were the off-latency, the on-latency, the conditioning-latency, and the off-/on-latency difference.

\section{Results}

The motor program which controls the specific EMG activities was translated into the gait dynamics of the $y$-axis vector of the sole. There were two modes of the motor programs controlling the synergy of gait (Fig. 1). The motor program as the mode or code of motor equivalence was dependent upon whether the gait was self-paced or triggered. The selfpaced gait mode was due to the motor program initiating the unilateral tibialis anterior EMG activity, and this EMG activity was associated with the unilateral backward bodysway of the same leg (Fig. 1a) (Elble 1997). The performance of triggered gait mode was triggered by the visual or auditory cue that served as the on-stimulus. The motor program which controls gait initiation showed a burst of tibialis anterior EMG activity bilaterally (on-latency) (Fig. 1b; no-gap-task). Bilateral EMG activity occurred nearly simultaneously, and was associated with the bilateral dynamic backward sway of the body, which connected to the steady stance/swing phase of the dynamic gait initiation in each leg. The gait modes triggered by the visual or auditory cue were essentially identical EMG and y-vector patterns (i.e., triggered gait mode).

We analyzed the motor program affecting the synergy of gait by controlling the triggered gait mode among the gap, overlap, and no-gap-tasks in the following study. The off-latency of the soleus EMG pause combined with the onlatency burst of tibialis anterior EMG activity was a setsynchronized response in normal subjects. About $50-80 \mathrm{~ms}$ before the burst, the soleus EMG tonic activity paused (offlatency) bilaterally in elderly control subjects (Fig. 1b). The interval between the off-latency soleus EMG pause and the on-latency burst of tibialis anterior EMG activity (off-/onlatency difference) and the $y$-axis vector pattern were identical among the gap, no-gap, and overlap-tasks in each subject (see below).

Figure 2 shows the results of each patient's performance ( $\mathrm{H}-\mathrm{Y}$ stages II and IV) during the gap-task. In the gap-task which was triggered solely by the on-stimulus, tibialis anterior on-latencies were statistically unchanged among stages II-IV patients and normal subjects (Fig. 2, Table 1). In the overlap-task which was triggered by both the on-stimulus and the remaining visual-conditioning-stimulus, both the offand on-EMG latencies were remarkably and simultaneously prolonged in patients with Parkinson's disease (Fig. 3). The two responses of the off- and on-latencies were dissociated between the gap and overlap-tasks in each subject. In the advanced stages, the off-latency was considerably prolonged, and the off-on-latency difference (interval) decreased or was negative in all three of the tasks (Figs. 2b, 3b). When all three tasks were performed by patients with advanced bradykinesia of slow gait, the tibialis anterior EMG activity reduced the amplitude. Soleus EMG activity reduced the 
Fig. 1 Two gait modes (selfpaced and triggered gait mode). Two different motor programs which control two gait modes (self-paced and triggered mode) were shown in each subject. Six gait trials of a normal subject were superimposed, and were aligned with the onset of the tibialis anterior burst of EMG activity (vertical broken line) in each gait mode. There were two modes of the motor programming in both the EMG activity and the dynamic $y$-axis vector. Self-paced gait mode (a); gait initiation started unilaterally with a unilateral tibialis anterior burst of EMG activity. Triggered gait mode (b); the gait initiation started bilaterally with the bilateral burst of EMG activities of tibialis anterior (triggered by a visual on-stimulus)

\section{a Self paced gait mode (Young)}

(Y21107)

$(18 \mathrm{M}, 4.0 \mathrm{~km} / \mathrm{h})$

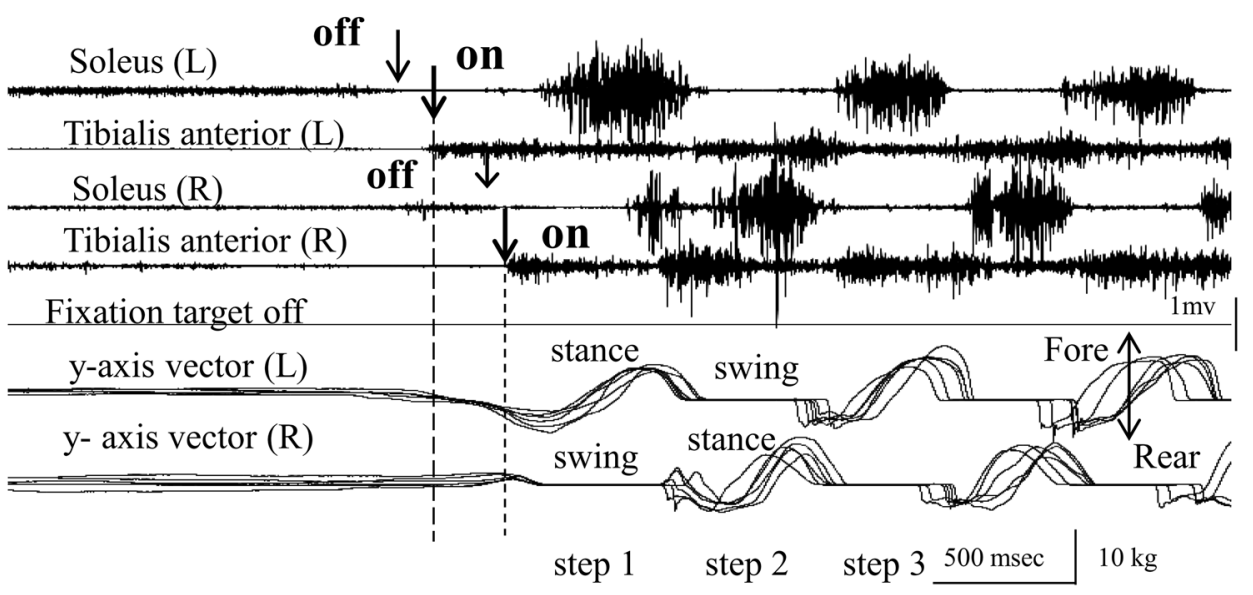

b Triggered gait mode (Eldely)

(E31106) $\quad(71 \mathrm{~F}, 3.2 \mathrm{~km} / \mathrm{h})$

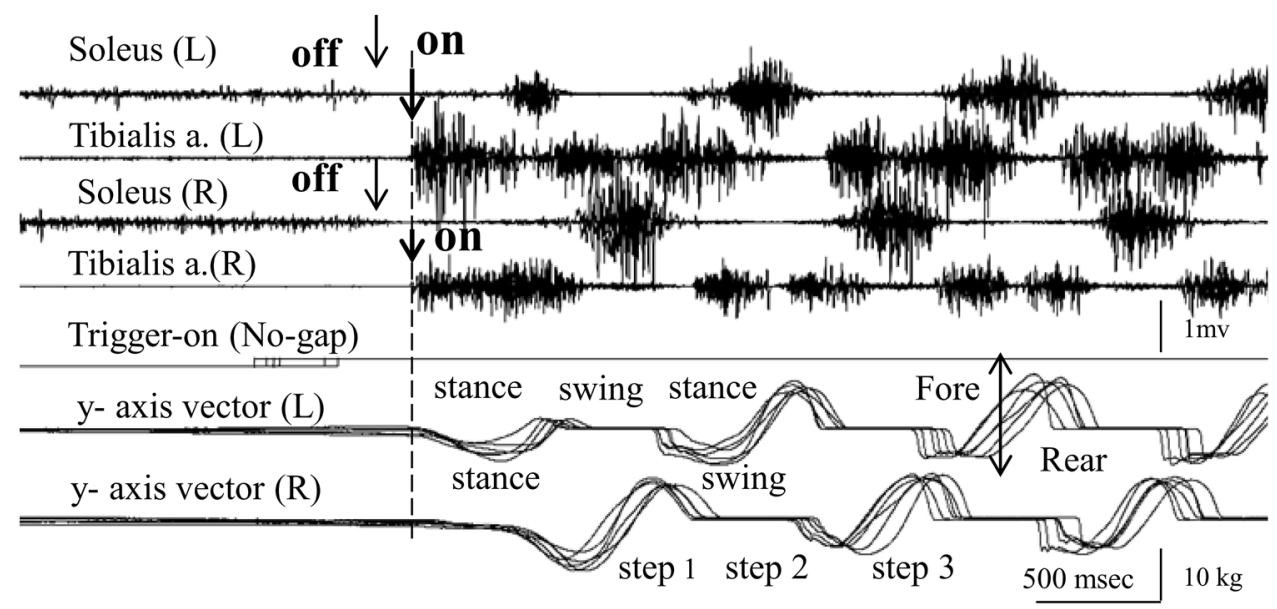

amplitude but approximated the long-lasting EMG activity, which was in contrast to the phasic EMG activity of the early stage (Figs. 2b, 3b).

Figure 4 plots the mean values of the latencies in all subject groups (stages II-IV of Parkinson's disease), including young and elderly normal subjects against the value of synchronization of the off-/on-latency difference in each subject group (inset figure keys). In the gap-task, the patients' initiation of gait was triggered without the visual-conditioningstimulus. The mean values of the soleus off-EMG latency were prolonged in association with the off-/on-latency difference (Fig. $4 \mathrm{~A}_{1}$ ). The tibialis anterior on-latency mean values were statistically identical in all subjects including

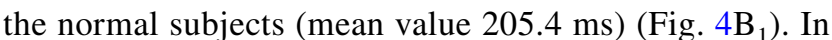
the advanced stages of Parkinson's disease, however, the responses between the off- and on-EMG latencies were dissociated. The off-/on-latency difference decreased with the advancement of the disease, and the off-/on-latency interval was unsynchronized (co-contraction) in the advanced stages of the disease. Figure 5 plots the mean latencies of the onset of the soleus pause and the onset of a tibialis anterior EMG burst. This is to the test stimulus during the gap, no-gap, and overlap-tasks ( $\mathrm{G}, \mathrm{Ng}$, and $\mathrm{Ov}$ ) against different subject groups. In contrast, conditioning- latencies which are the latency differences between the overlap and gap-tasks $(\mathrm{Ov}-\mathrm{G})$ were prolonged in association with the advancement of the disease. Conditioning-latency (Fig. 4A, $\mathrm{B}_{2}$ ): we examined the effect of the visuospatial fixation target on the off- and on-latencies in the overlap-task. The off- and on-latencies which represent the conditioning-latencies were nearly identical. The conditioning-latencies of the visuospatial cognitive sensory-motor programming were prolonged in association with the off-/on-latency difference and the advanced Hoehn and Yahr stage of Parkinson's disease (Figs. 4A $2, \mathrm{~B}_{2}, 5$ ).

The dynamics of the $y$-axis vector pattern were basically similar among the three tasks in each patient. The dynamic form of gait changed in the advanced stages of Parkinson's 
Fig. 2 Motor programming of the gap-task in stages II and IV Parkinson's disease. The gap-task is solely based on the trigger on-stimulus without the conditioning-stimulus of a fixation target. Six gap-task performances of a patient's leg EMG activities and the $y$-axis vectors were superimposed and aligned with the onset of tibialis anterior EMG in either stage II (a) and stage IV patient (b). The on-latency was statistically identical, but the off-latency in the advanced stage IV patient prolonged the latency more than the on-latency. The off-/ on-latency interval (difference) was about $50 \mathrm{~ms}$ in the stage II patient (a), but it was negative in the stage IV patient (b). It is noted in stage IV that the soleus EMG off-latency was prolonged and the tibialis anterior EMG activity reduced (b). In the stance phase of stage IV patient, the backward bodysway decreased remarkably and the stance phase gait dynamics changed to the forward bodysway (unsteady walking on one's tiptoe). The swing phase (zero-flat level of $y$-axis vector) decreased in the advanced stage patient (b)
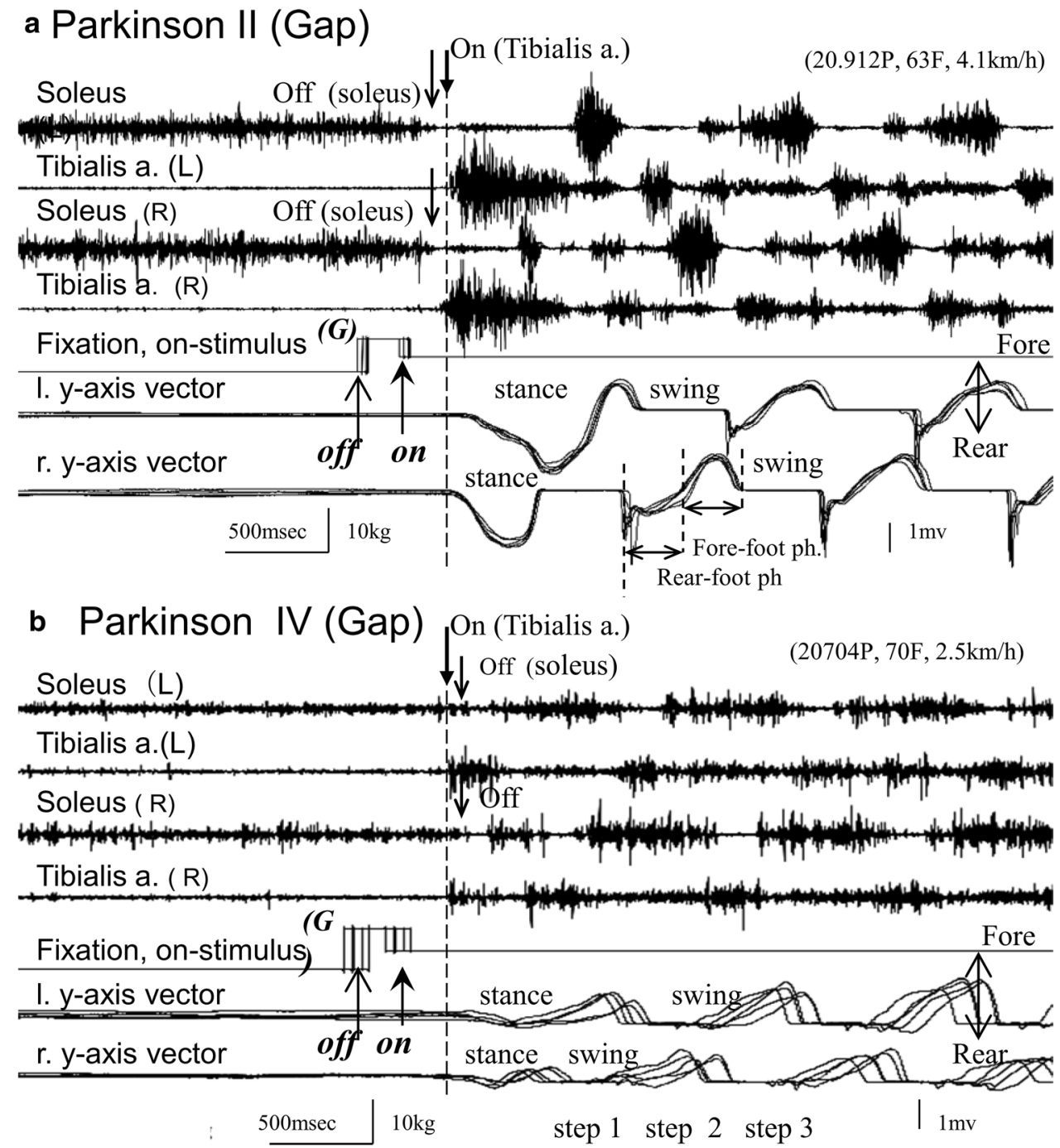

disease, but the stance phase durations were virtually identical (Fig. 6a). The stance phase is formed from the rear-foot phase and the fore-foot phase (inset figure in Fig. 6a). The rear-foot phase (backward body-sway as the leading gait dynamic) of the initial stance phase was decreased bilaterally in duration and amplitude in the majority of patients in the advanced stages of Parkinson's disease (Figs. 2b, 3b). In contrast to the decreased duration of the rear-foot phase, the duration of the fore-foot phase increased in the stance phase, which included a forward leaning posture and the shuffling-step gait which is commonly seen in the disease. We examined the four steps after the third step in each patient and control subject. The mean \pm SD ratios of fore-foot duration over the stance phase duration during the third-sixth steps were $50.1 \pm 3.2 \%$ in the elderly controls $(n=9), 54.1 \pm 6.0 \%$ in the stage II patients $(n=11), 63.9 \pm 6.8 \%$ in the stage III patients $(n=13)$, and $77.7 \pm 7.1 \%$ in the stage IV patients $(n=11)$.

Figure 6a plots the mean duration of each swing/stance phase of the six steps after the third step for all subject groups. The swing phase duration shortened in all steps, and decreased more in stages III and IV (Fig. 6a). The step gain of gait decreased in the advanced stages of Parkinson's disease (Fig. 6b). Patients with H-Y stages III and IV (but not II) exhibited decreased step gain in all six steps (Fig. 6b). The step gain was decreased, but unchanged among the three tasks in the advanced stages of Parkinson's disease. The selfpaced walking speeds decreased in the advanced stages of the disease.

Figure 7 plots the off-/on-latency difference of synergicgait movement in all of the patients with Parkinson's disease, including the nine elderly control subjects (keys), against their gait speed during the no-gap-task. The off/on-latency difference correlated with the speed of the synergic-gait movement of Parkinson's disease including the elderly control. There was a significant correlation between the two as a fitted logarithmic function. The regression line and the coefficient of correlation was calculated as $F(x)=57.97$ $\log \mathrm{x}-21.64(p<0.0001)$. The decreased gait speed of 
Table 1 Off-/on-mean-latency and step gain in different subject group

\begin{tabular}{|c|c|c|c|c|c|c|c|c|c|c|}
\hline \multirow[t]{2}{*}{ Latency (ms) } & \multicolumn{2}{|c|}{ Parkinson II (11) } & \multicolumn{2}{|c|}{ Parkinson III (13) } & \multicolumn{3}{|c|}{ Parkinson IV (11) } & & \multicolumn{2}{|c|}{ Elderly control (9) } \\
\hline & Mean & SD & Mean & SD & & Mean & SD & & Mean & SD \\
\hline \multicolumn{11}{|l|}{ Soleus off } \\
\hline Overlap & 235.5 & 35.9 & 278.8 & 52.7 & & 348.4 & 65.5 & & 199.9 & 47.4 \\
\hline No-gap & 185.4 & 37.1 & 219.5 & 54.3 & & 253.3 & 48.0 & & 173.0 & 37.6 \\
\hline Gap & 159.7 & 40.7 & 189.8 & 41.5 & & 214.3 & 37.7 & & 147.6 & 37.2 \\
\hline \multicolumn{11}{|l|}{ Tibialis A on } \\
\hline Overlap & 275.1 & 28.1 & 299.5 & 48.2 & & 343.4 & 55.8 & & 253.9 & 50.3 \\
\hline No-gap & 230.1 & 36.3 & 241.3 & 54.1 & & 249.7 & 53.2 & & 227.5 & 37.9 \\
\hline Gap & 202.7 & 41.8 & 209.5 & 37.7 & & 206.7 & 37.0 & & 204.9 & 37.9 \\
\hline \multicolumn{11}{|l|}{ Off-on latency difference } \\
\hline Overlap & 39.6 & 14.2 & 20.6 & 13.6 & & -4.9 & 14.3 & & 53.9 & 6.5 \\
\hline No-gap & 44.6 & 8.4 & 21.8 & 8.0 & & -3.5 & 16.5 & & 54.5 & 6.2 \\
\hline Gap (3-6th step) & 43.0 & 11.2 & 19.7 & 13.5 & & -7.5 & 18.0 & & 57.3 & 9.8 \\
\hline \multicolumn{11}{|l|}{ Step gain $(\mathrm{Sw} / \mathrm{ST})$} \\
\hline Overlap & 0.555 & 0.066 & 0.441 & 0.063 & & 0.387 & 0.058 & & 0.562 & 0.070 \\
\hline No-gap & 0.551 & 0.070 & 0.431 & 0.051 & & 0.387 & 0.058 & & 0.575 & 0.068 \\
\hline Gap & 0.554 & 0.066 & 0.438 & 0.065 & & 0.390 & 0.059 & & 0.573 & 0.074 \\
\hline \multirow[t]{2}{*}{ Two-way ANOVA } & \multicolumn{3}{|c|}{$\begin{array}{l}\text { Task conditions (A) (overlap no-gap, } \\
\text { gap) }\end{array}$} & \multicolumn{4}{|c|}{$\begin{array}{l}\text { Subject groups (B) (elderly vs. Par- } \\
\text { kinson) }\end{array}$} & \multicolumn{3}{|c|}{ Interaction $(\mathrm{A} \times \mathrm{B})$} \\
\hline & FA & $d f$ & $p$ & FB & $d f$ & $p$ & & FAB & $d f$ & $p$ \\
\hline Soleus off-latency & 41.0426 & 2 & $<0.0001$ & 26.5306 & 3 & & & 1.4934 & 6 & 0.1861 \\
\hline Tibialis A on-latency & 43.0250 & 2 & $<0.0001$ & 4.1518 & 3 & & & 1.8082 & 6 & 0.1031 \\
\hline Off-on latency difference & 0.2946 & 2 & 0.7454 & 141.084 & 3 & & & 0.2420 & 6 & 0.9616 \\
\hline Step gain & 0.0710 & 2 & 0.9315 & 62.2771 & 3 & & & 0.0808 & 6 & 0.9979 \\
\hline
\end{tabular}

Mean \pm SD of off- and on-latencies are summarized in the gap, no-gap, and overlap- tasks (G, Ng, Ov) against different subject groups

Two-way ANOVA analysis for soleus off-latency indicated that all factors of task conditions (gap, no-gap, overlap) and subject groups (elderly controls, Hoehn and Yahr stages II-IV of Parkinson's disease) showed a significant difference $(p<0.0001)$ but without significant interaction between the task and the subject

bradykinesia in Parkinson's disease showed a significant correlation with the unsynchronized synergic-gait movement (Fig. 7). There was no significant difference in each subject's gait speed during the three tasks. The mean \pm SD were $3.2 \pm 0.7 \mathrm{~km} / \mathrm{h}$ in the elderly control, $3.0 \pm 0.6 \mathrm{~km} / \mathrm{h}$ in stage II $2.3 \pm 0.6 \mathrm{~km} / \mathrm{h}$ in stage III, and $2.0 \pm 0.5 \mathrm{~km} / \mathrm{h}$ in stage IV in no-gap-task.

Table 1 summarizes the mean \pm SD off-latencies during the three task conditions in different subject groups. Twoway ANOVA analysis for soleus off-latency indicated that all the factors regarding the task conditions (gap, no-gap, and overlap) and subject groups (elderly controls, $\mathrm{H}-\mathrm{Y}$ stages II-IV) showed significant differences $(p<0.0001)$, but without any significant interaction between the task and the subject. Two-way ANOVA analysis for tibialis anterior on-latency indicated that there was a significant difference in both task conditions $(p<0.0001)$ and subject groups $(p=0.0077)$ without any significant interaction between the two. Two-way ANOVA analysis of the off-/on-latency differences indicated that there was a significant difference among subject groups $(p<0.0001)$, but no significant difference in the task conditions. There was no significant interaction between the two (Table 1). We also performed an ANOVA analysis on step gain (Table 1); there was a significant difference in subject groups, $(p<0.0001)$ but no significant difference in task conditions. There was no significant interaction between the two.

\section{Discussion}

The previous studies concerning possible impairment of the switching process in patients with Parkinson's disease examined heterogeneous sequential movements (Agostino et al. 1992; Benecke et al. 1987; Harrington and Haaland 1991). However, these impaired switching processes indicated the general deficits of the capacity to switch within heterogeneous sequential on-on reaction time, but disregarded EMG 
Fig. 3 Motor programming of the overlap-tasks in stages II and IV Parkinson' disease. Six overlap-tasks of a patient's leg EMG activity and the $y$-axis vectors were superimposed and aligned with the onset of tibialis anterior EMG in either stage II (a) or stage IV patient (b). In the overlap-task, the on-stimulus was turned on before the primary fixation-stimulus was turned off (200 ms-overlap). In the overlap-task, we examined the visual cognitive conditioning-stimulus as the environment effects on the EMG latencies. Both the off- and on-latencies of leg EMGs were prolonged in stage IV. The off-/on-latency difference (interval) was about $50 \mathrm{~ms}$ in the stege II patient (a). In the stage IV patient (b); each mean value off-/on-latencydifference was unsynchronized or negative. In stage IV (b), the tibialis anterior EMG activity was reduced, but the soleus EMG activity lengthened the tonic EMG activity, and the stance phase $y$-axis vector changed to the forward dynamic body-sway

\section{a Parkinson II (Overlap task)}

$(\mathrm{P} 20912,63 \mathrm{~F}, 4.1 \mathrm{~km} / \mathrm{h})$

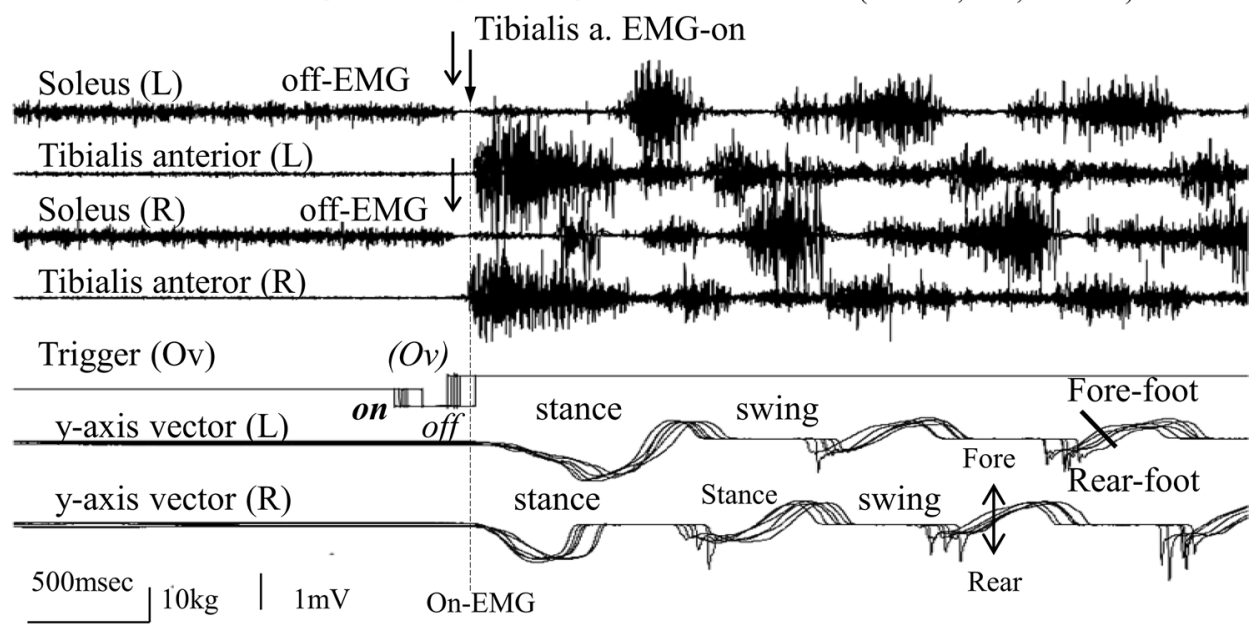

b Parkinson IV (Overlap task)

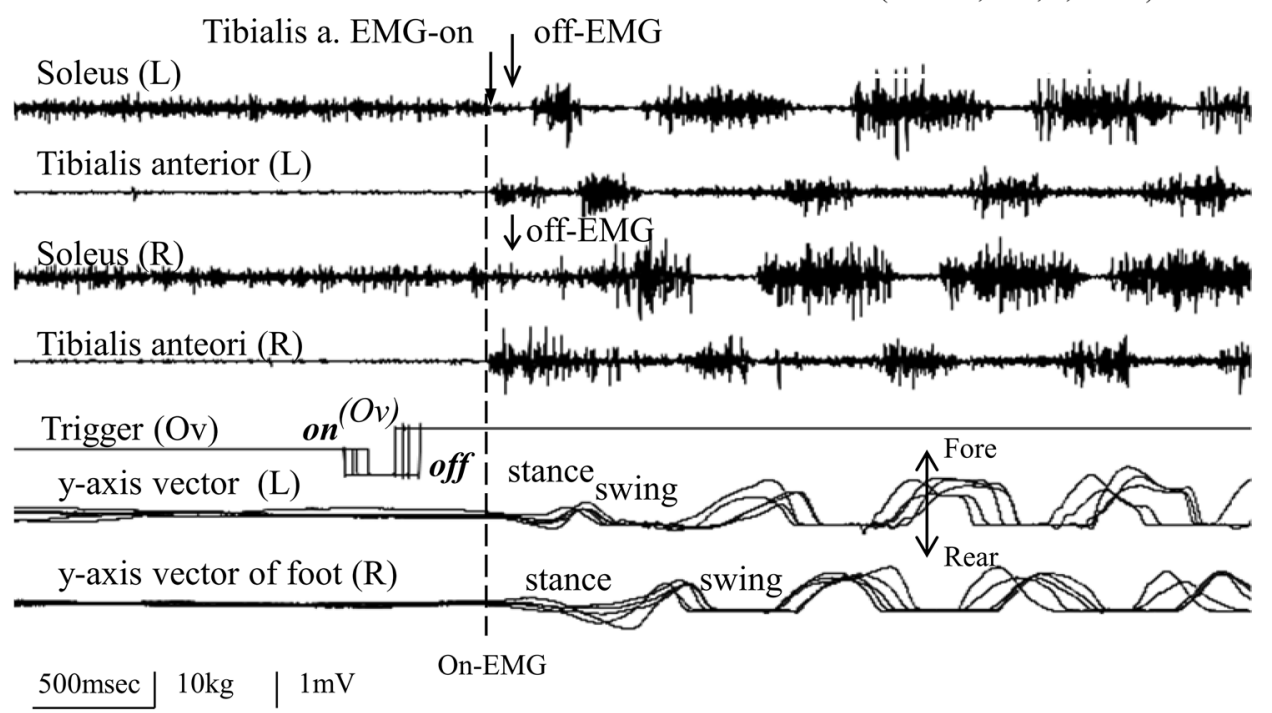

off-responses. In contrast, the dynamic gait initiation indicated that the termination/initiation between the two antagonist muscles is dissociated and the two motor programs function independently. These results changed the evaluation of the sensory-motor process of gait bradykinesia.

\section{Off-latency}

The dynamic gait initiation is controlled by the successively synchronized antagonist EMG activity which is seen in the termination/initiation process. This activity involves the suppression (pause) of the soleus tonic EMG activity and the burst of EMG activity from the tibialis anterior (Basmajian 1974; Carpinella et al. 2007). Our experimental paradigms facilitate dissociation of responses between the off- and on-latencies, indicating that the off- and on-latencies are controlled independently by sensory-motor programs in Parkinson's disease (Fig. 2, Table 1).

In general, the movement disorders in Parkinson's disease have been regarded as resulting from imbalances in the direct, indirect, and hyperdirect pathways of the basal ganglia (Crutcher and DeLong 1984; Leblois et al. 2006; Mink 1996). Experimental studies indicated that the direct pathway connects mono-synaptically from the striate neurons to the $\mathrm{GPi} / \mathrm{SNr}$, which promote the intended primary motor programs and context (Mink 1996; Nambu et al. 2002a, b). In contrast, the indirect pathway projects poly-synaptically to the GPi/SNr by way of sequential connections with the GPe and STN, which suppress unnecessary motor programs (Mink 1996; Nambu et al. 2002a, b).

Nambu (2008) emphasized the mechanism of a 'hyperdirect' pathway projecting to the STN as the collateral of pyramidal tract neurons, which cooperate with indirect and 

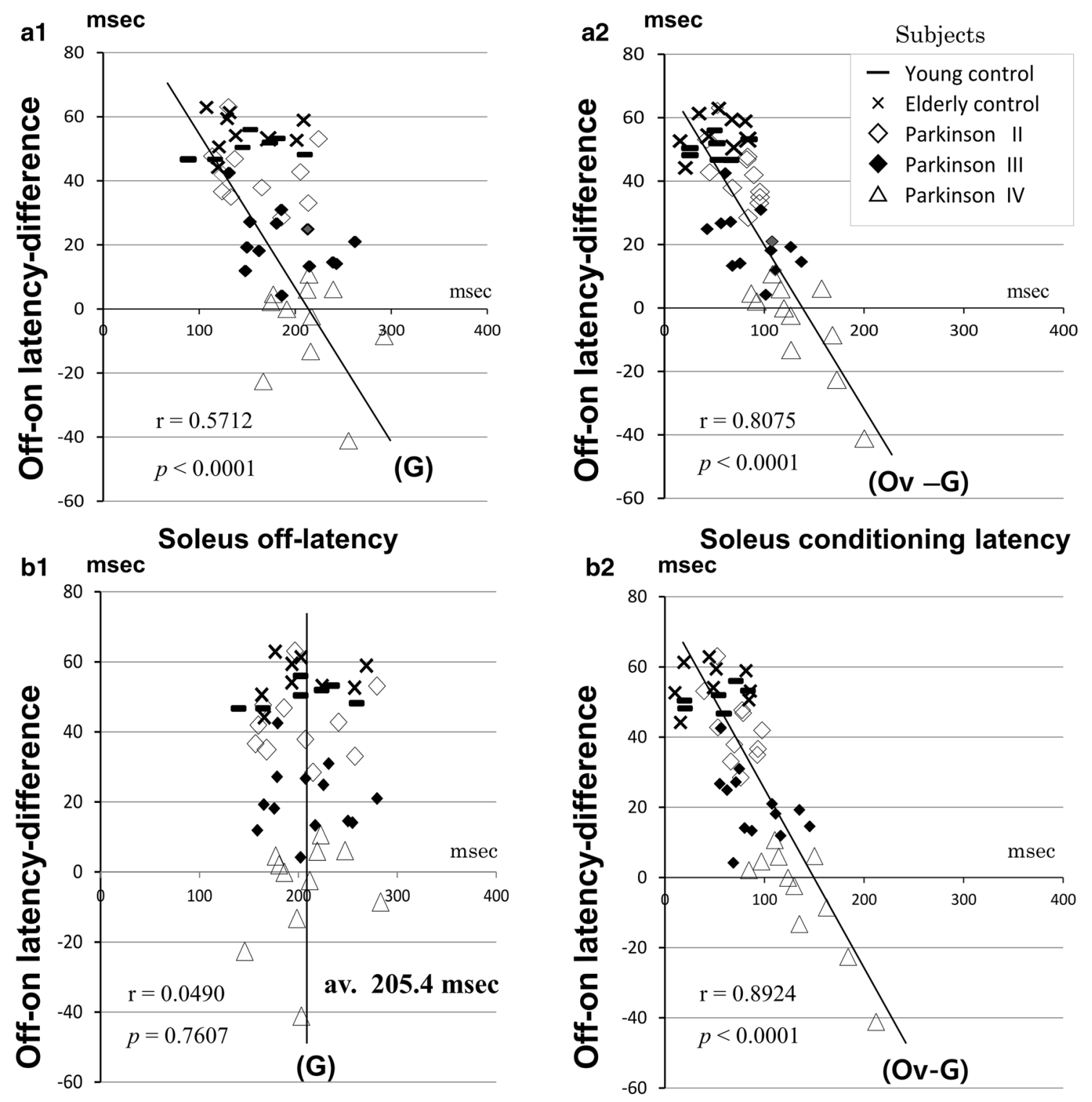

Tibialis A. on-latency

Fig. 4 Off-latency, on-latancy and conditioning-latency against to the off-/on-latency difference of soleus and tibialis anterior EMG. The off-/on-latency difference (ordinate) decreased in the advancement of the disease among the subject groups (dash, times, open diamond, filled diamond, open triangle; keys). $\mathbf{A}_{1}$ plots the mean latency of the soleus off-EMG latency against the off-/onlatency-difference (interval) during the gap-task $(\mathrm{G})$. The mean values of the soleus off-latencies were prolonged in the advanced stages of the disease. $\mathbf{B}_{\mathbf{1}}$ plots the mean latency of the tibialis anterior on-

direct pathways. His interpretation is that as the established function, the hyperdirect pathway terminates competing motor program and organizes the next motor program in GPi/SNr. Thus, GPe and STN establish the conversion process from the competing motor program to the focally selected motor program in GPi/SNr (Mink 1996; Nambu
2008). Subsequently, the uncompetitive organized motor programs persist in GPi/SNr (Mink 1996; Nambu et al. 2002a; Nambu 2008).

The triggered dynamic gait initiation from an upright posture results in a loss of balance due to of the cessation of voluntary tonic EMG activity in the soleus and postural 


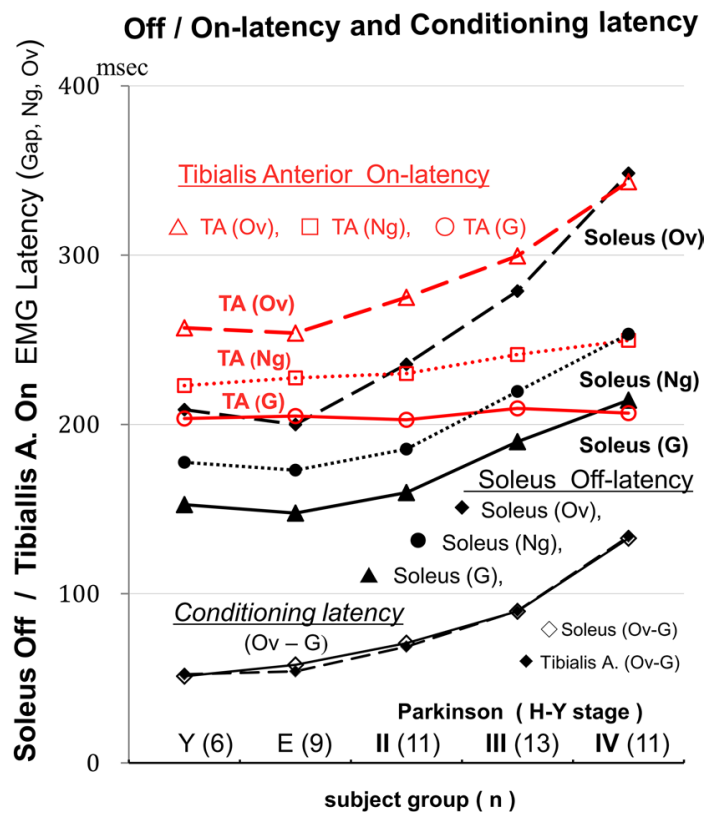

Fig. 5 Tibialis anterior on-latency and soleus off-latency against different subject groups of young, elderly control, and H-Y stage-II, stage-III, and stage-IV, which were in each task of gap, no-gap, and overlap. The mean soleus off-latencies (black line, filled diamond, filled circle, filled triangle) and the mean tibialis anterior on-latencies (red line, open triangle, open square, open circle) are plotted during the gap, no-gap, and overlap-tasks (G; solid line, $\mathrm{Ng}$; dotted line, Ov; broken line) against different groups. It is notable that the on-latencies of the gap-task are statistically unchanged in all subjects. Both the off- and on-latencies were prolonged in the overlap-task. The conditioning-latency (effect) in the overlap-task which is regarded as the latency difference of the two tasks $(\mathrm{Ov}-\mathrm{G})$ is plotted in each off- and on-latency (off-latency; open diamond, on-latency; filled diamond). The prolongation of the off-latency and the conditioning-latency were correlated with the advancement of Parkinson's disease. The off-/onlatency-difference (interval) decreased with the advancement of the disease (between the red line and the black line in each task) muscles (Basmajian 1974; Carlsöö 1966). This study confirms that the termination and cessation in the voluntary tonic EMG activity of the soleus muscle is difficult in Parkinson's disease. Crenna et al. (2006) indicated that the STN stimulation changed the start of gait dynamics. The clinical study in patients with Parkinson's disease indicated that the STN stimulation played a role in the termination of voluntary movement (Hsu et al. 2012). The results of the present study, however, indicated that the decreased gait speed of bradykinesia correlates with the unsynchronized off-/onlatency interval, which is due to the excessive long tonic EMG activity of the soleus muscle in the advanced stages of the disease (Fig. 7). Furthermore, in these results, the leading gait dynamics of the stance phase changed from the backward body-sway to the forward body-sway (Figs. 2b, $3 b)$ and the swing phase was shortened due to the observed decrease of EMG activity from the tibialis anterior muscle in the advanced stages of the disease (Fig. 6b). Thus, it was suggested that the difficulty in terminating the competitive motor programs which are controlling the primary postural soleus muscle is one of the major disorders in the bradykinesia of Parkinson's disease.

However, it was assumed that before the termination of the competitive motor program, the motor program engages in the determination of whether the gait mode is self-paced or triggered (Fig. 1). In the triggered gait mode, the primary posture was terminated bilaterally and the backward leadingdynamic change of gait initiation was started bilaterally. In the self-paced gait mode, the posture was terminated unilaterally, and the leading dynamics were started on the same side. These results suggested that the STN and GPi, through the collateral of the pyramidal tract, also contribute to the gait mode (Fig. 8).
Fig. 6 Changes of swing phase duration and step gain in Parkinson's disease. Figure 6a plots the mean duration of the stance phase and the swing phase in each of the first six steps in the young controls $(n=6)$, the elderly controls $(n=9)$, and the patients with Parkinson's disease $(n=35)$ in the no-gap-task condition. The swing phase decreased with the advancement of the disease, but the stance phases were nearly identical among the different subject groups (keys). Figure 6b plots the mean step gain in each of the first six steps in different subject groups (keys). For further explanation, see the text
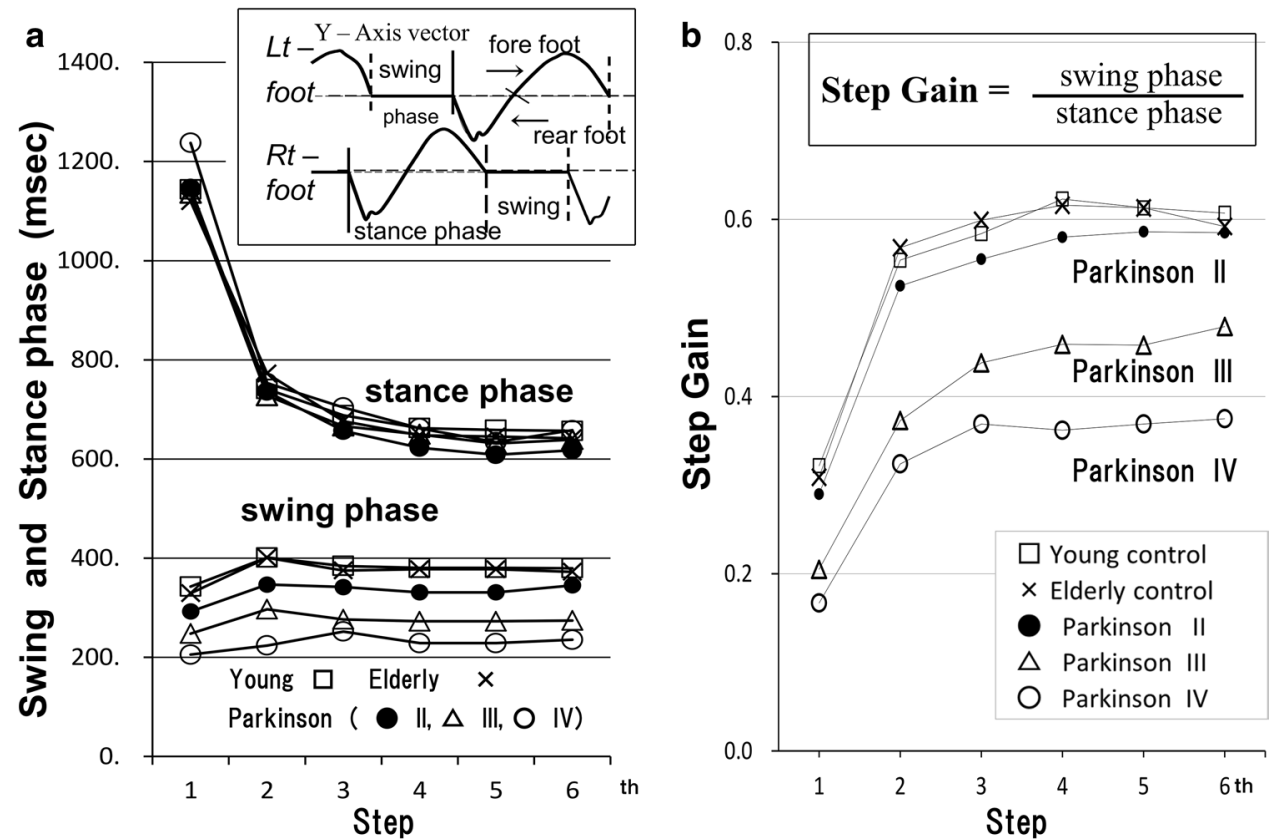


\section{Slowness of synergic gait movement}

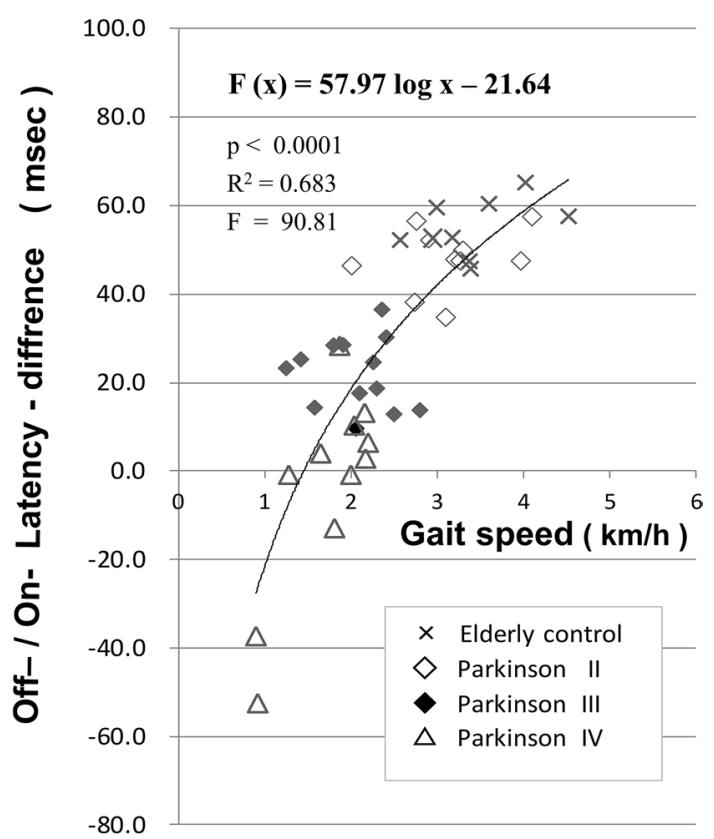

Fig. 7 Gait slowness and the un-synchronous off-/on-latency motor programming. The gait speed of synergic movement decreased in the advanced stages of bradykinesia as seen in Parkinson's disease. The gait speed was plotted against the off-/on-latency-difference in the patients with Parkinson's disease including the normal elderly control subjects (keys). The off-/on-latency difference was short or negative in the advanced stages of the disease. Gait speed $(\mathrm{km} / \mathrm{h})$ in Parkinson's disease was correlated exponentially with the off-/on-latency difference $(p<0.0001)$. It was noted that the gait bradykinesia is involved in the unsynchronized off-/on-latency, which indicated the difficulty in terminating the prior posture/movement. The gait bradykinesia of synergic movement was regulated by two independent motor programs of the off- and on-latency levels. The short or negative off-/on-latency difference indicated the off-/on-latency levels of sensory-motor programs are not synchronizing in the advanced stages of Parkinson's disease

\section{On-latency}

Anatomically, striatal neurons related to movement receive input from the somatosensory and motor cortex with a topographic relationship between the cerebral cortex and the striatum (Agostino et al. 1992; DeLong and Wichman 2007; Mink 1996; Nambu et al. 2002b). In addition, the basal ganglia circuitry is composed of direct and indirect dopaminergic pathways, which are related to the organizing context of overall synergic movements (Alexander and Crutcher 1990; Crutcher and DeLong 1984; DeLong and Wichman 2007). Based on these anatomical and physiological relationships, the information from the basal ganglia returns to the cerebral cortex via the thalamus through the cortico-basal ganglialoop and controls the neural activity of the cerebral cortex in the voluntary synergic movement (Alexander et al. 1986; Leblois et al. 2006; Nambu et al. 2002a).
However, studies using trained monkeys showed that most of the basal ganglia cells activity had begun after the onset of EMG activity and before the onset of new movement (Crutcher and DeLong 1984; Crutcher and Alexander 1990; Georgopoulos et al. 1983; Wichman et al. 1994). These experimental results indicated that the basal ganglia are unlikely to be the initial motor command signal. The neural activity in the direct pathway, however, is related to the dimensionally past-time uncompetitive selected motor program in the basal ganglia, which may pursue the ongoing dynamic synergic movement as the on-latency in the cerebral cortex. Furthermore, the motor program of the offlatency system is dimensionally the past-time competitive motor program, which may terminate via the basal ganglia in contrast to the on-latency on-going motor program in the cerebral cortex (Fig. 8; diagram modified from Nambu et al. 2002b).

The results of the present study suggested that the dynamic gait initiation is controlled by two independent levels of termination/initiation motor mechanisms as the off- and on-latency motor program. In healthy subjects, the past-time off-latency motor program and the on-going onlatency motor program were synchronized successively in the initiation of gait. However, in Parkinson's disease, the off-latency motor program connected to the previous posture prolonged the competitive activity (latency), and the off-latency and on-latency motor programs were unsynchronized during the initiation of gait.

On the other hand, the basic nervous mechanisms constituting the locomotor pattern generator are located the cerebral cortex, the brain stem, and the lumbosacral spinal cord (Dietz et al. 1995; Takakusa 2013), while recent researchers have pointed out anatomically direct mono-synaptic connections from the pyramidal tract to the moto-neurons innervating the lower leg muscles of humans (Maertens de Noordhout et al. 1999; Jahn et al. 2008a, b). Bawa et al. (2002) revaluated the nature of coritico-moto-neuronal connections onto tibialis anterior and soleus moto-neurons in human subjects, and found that all ankle flexor moto-neurons receive strong cortico-moto-neuronal connections, but the soleus receive very few.

Cioni et al. (1997) indicated that the EMG activity of the tibialis anterior muscles was reduced during steadystate walking in Parkinson's disease. In the present study of advanced cases of Parkinson's disease, the tibialis anterior EMG activity reduced the amplitude, and soleus EMG activity reduced the amplitude and approximated to the longlasting EMG activity. Furthermore, in gait initiation, these results indicated that the on-latency of the tibialis anterior is unchanged in all subjects including the normal subjects, during the gap-task. The authors conjectured that the on-latency was identical due to the reply form the cortical selected motor program's direct connection from the pyramidal tract 


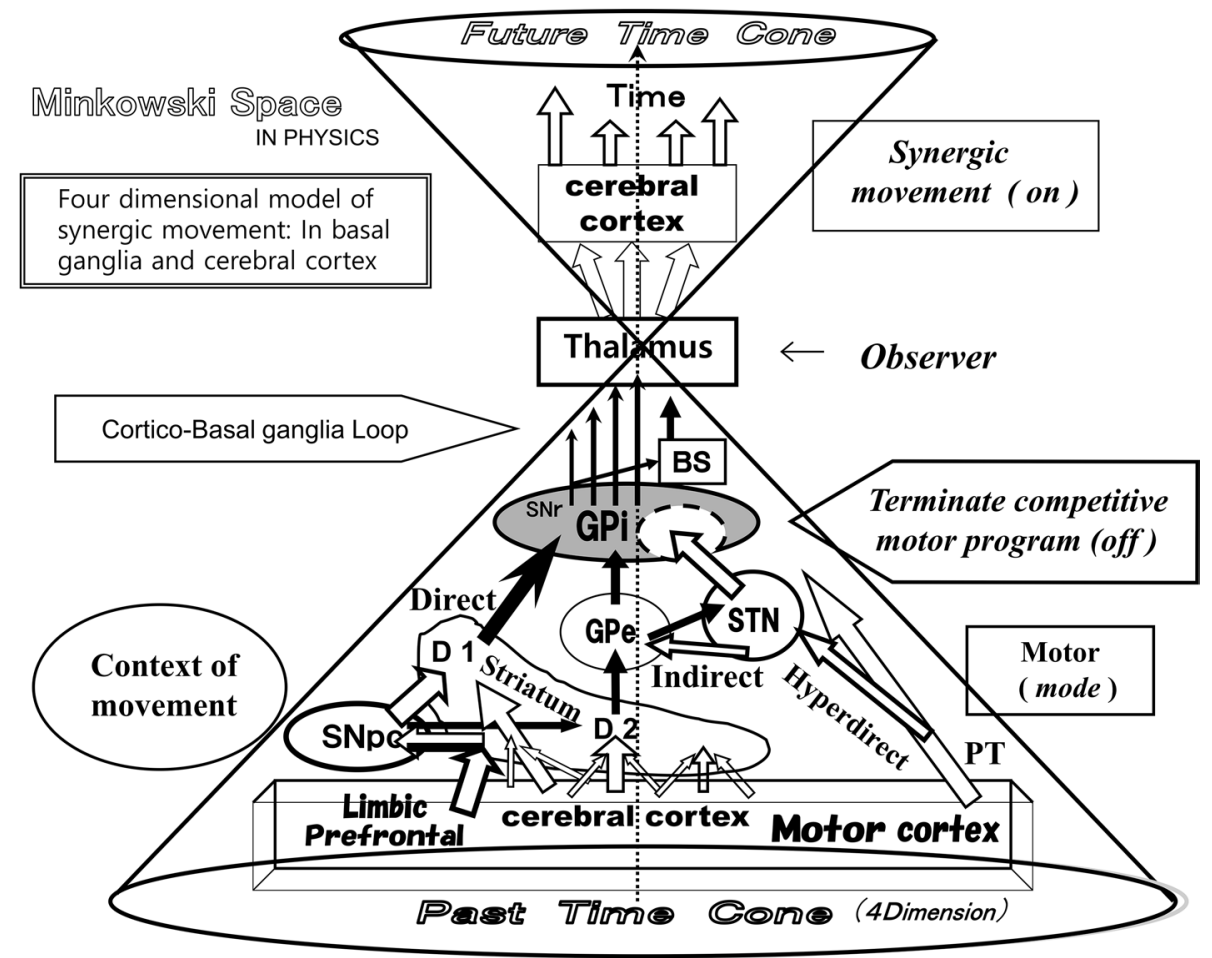

Fig. 8 Four-dimensional image model of the basal ganglia (Minkowski space in where space and time are interwoven in physics). We hypothetically propose that the model of synergic movement in Parkinson's disease can be viewed as the four-dimensional coordinate in physics of time and space (Minkowski 1908; Sard 1970). This four-dimensional image model was modified from the basal ganglia anatomy of Nambu et al. (2002b). The ordinate represents time (past/future; down/up). The abscissa represents the three-dimensional space schematically. Programs of foregoing and on-going synergic movement were carried from the cerebral cortex to the striatum

(Bawa et al. 2002). During the gap-task, this was observed in all of the subjects.

\section{Overlap-task}

Numerous studies have indicated that the difficulty of gait initiation is related to the visuospatial cognitive situation in Parkinson's disease (Azulay et al. 1999; Cummings and Steven 1992; Low et al. 2002; Naismith et al. 2010; Nantel et al. 2012; Rodriguez-Oroz et al. 2001). In this study, the residual visual-conditioning-stimulus (200 ms), which is competing with the on-stimulus in the overlap-task simulates the visuospatial cognitive situation. The values of the conditioninglatencies (effects) were statistically identical in both the off-latency and on-latency systems in the overlap-task, and prolonged in parallel in accordance with the progression of the disease (Table 1). Hence, regarding the visual-conditioning-stimulus, we hypothesized that the parallel prolongation of the conditioning-latency involves a common anatomical (lower past-time cone). To perform the successive synergic movement (on-latency), the previous uncompetitive motor program recurs through the cortico-basal ganglia feedback loop of the basal ganglia (GPi), which anatomically shares a connection with the organized output of cerebral cortex via the basal ganglia. In Parkinson's disease, however, the unsynchronized motor programming was characterized by the difficulty in terminating the foregoing competitive motor program (off-latency) via the STN and GPe. For further explanation, see the text

pathway, which activates both the off- and on-latency system in the basal ganglia in parallel (Fig. 8).

Anatomically, striatal neurons receive input from the somatosensory and the motor cortex (Agostino et al. 1992; Georgopoulos et al. 1983; Nambu et al. 2002b). The GPe neurons activity receives the cortical output through the indirect pathway (D2) of the dopaminergic system in striatum which is connected in parallel with both the STN and the GPi neurons (Crutcher and DeLong 1984; DeLong and Wichman 2007; Rodriguez-Oroz et al. 2001). Referring to these anatomical pathways, the cognitive signal of the conditioning-stimulus connecting to the dopaminergic system may be returning to the cerebral cortex through the cortico-basal ganglia parallel loop. The results documented in this paper indicate that the pathologically parallel delayed off-/on-latencies are due to the difficulty in terminating the sensory-motor programming of the visuospatial conditioning in the basal ganglia (Fig. 8) of patients with Parkinson's disease. 
The conditioning-stimulus effect is different between the heterogeneous sequential motor programming of eye-hand coordination in Parkinson's disease (Warabi et al. 1986, 2011). These experimental results indicated that the development of bradykinesia with regard to the conditioningstimulus is not synchronous among the heterogeneous motor systems, which emphasized the bradykinesia of Parkinson's disease (Warabi et al. 1986).

\section{Synergic movement and gait dynamics}

Synergic movements usually start with the onset of a variety of successive EMGs, which are recruited in the trunk, thigh, arm, and other muscles prior to the initiation of movement (Basmajian 1974; Brain and Walton 1969; Brodal 1981). In this present study, the normal synergic movement was regulated by a pause of a 50-80 ms interval between the off- and on-latencies in the gait initiation of normal subjects (Fig. 1b). In the advanced stages, the tibialis anterior EMG activity decreased and the rhythmical phasic soleus EMG activity changed to nearly tonic EMG activity during successive walking in stages III-IV patients (Figs. 2b, 3b). Two antagonist muscles co-contracted and decreased the duration of the swing phase. Therefore, the step gain of gait was decreased in the patient with the pause of a short or negative off-/on-latency interval (Fig. 6b, Parkinson's disease of H-Y stages III, IV). Furthermore, Fig. 7 indicated that the gait speed of bradykinesia is correlated with the unsynchronized EMG activity of antagonist muscles in the leg (Figs. 2b, 3b). The gait speed correlation with the unsynchronized EMG activity changed in association with the progression of the disease, which was also related with the dopamine therapeutic effects on the symptoms. We conjectured that the unsynchronized antagonist muscles change the dynamic gait to the gait bradykinesia, which is due to difficulty in terminating the motor programming of the stance phase in the advanced stage of Parkinson's disease.

Anatomically, the information from the basal ganglia returns to the cerebral cortex via the thalamus through the cortico-basal ganglia-loop (Alexander et al. 1986; Leblois et al. 2006; Nambu et al. 2002a). Movement disorders including bradykinesia have been regarded as a reduced level of cortical motor output activity. The motor command output signal of dynamic gait initiation is organized not only by the cortical neurons, but is also recruited by the signal from the feedback loops resulting in a connection with the dopaminergic pathways of the basal ganglia (Alexander et al. 1986; Alexander and Crutcher 1990; DeLong and Wichman 2007). Leblois et al. (2006) suggested that these feedback loops are selecting the motor programs. The results of this present study suggested that the dynamic disorder of gait slowness is involved typically in the difficulty of terminating the prior sensory-motor program via the feedback signals from the basal ganglia (Fig. 8). The movement disorders in Parkinson's disease including bradykinesia have been thought to be due to the reduced cortical motor output activity (Wichman et al. 1994; DeLong and Wichman 2007; Nambu 2008). We estimated that the centrally organized brain output of the cerebral cortex changes the synergy of gait as the speed of a voluntary movement's ability to share a connection with the feedback signals from the basal ganglia.

\section{Conclusions}

(1) The synergy of gait was regulated by two independent motor programs of the off- and on-latency levels. (2) Delayed gait initiation of synergic movement in Parkinson's disease was due to difficulty in the termination of the sensory-motor program (fixation) which is linked with the cognitive visuospatial condition. (3) The slowness of the synergy of gait in Parkinson's disease was involved in the difficulty (long off-latency) in terminating the prior motor program which had been controlling the posture/movement.

Acknowledgements The authors express their gratitude to Prof. Astushi Nambu MD, PhD, for his valuable comments, and appreciate the English language advice provided by Prof. Mariya A. Niendorf $\mathrm{PhD}$, and Prof. Peter M. Olley MD, PhD. The technical assistance of EMG and $y$-axis vector analyses which were performed on Windows computer (Microsoft Excel) by Ms. Yukie Sasaki and Ms. Katelyn Funkhouser are greatly acknowledged.

Open Access This article is distributed under the terms of the Creative Commons Attribution 4.0 International License (http://creativecommons.org/licenses/by/4.0/), which permits unrestricted use, distribution, and reproduction in any medium, provided you give appropriate credit to the original author(s) and the source, provide a link to the Creative Commons license, and indicate if changes were made.

\section{References}

Agostino R, Berardelli A, Formica A, Accornero N, Manfredi M (1992) Sequential arm movements in patients with Parkinson's disease, Huntington's disease and dystonia. Brain 115:1481-1495

Alexander GE, Crutcher MD (1990) Functional architecture of basal ganglia circuits: neural substrates of parallel processing. Trends Neurosci 13:266-271

Alexander GE, DeLong MR, Strick PL (1986) Parallel organization of functionally segregated circuit links basal ganglia and cortes. Annu Rev Neurosci 9:357-381

Azulay JP, Mesure S, Amblard B, Sangla I, Pouget J (1999) Visual control of locomotion in Parkinson's disease. Brain 122:111-120

Basmajian JV (1974) Muscles alive: their functions revealed by electromyography, 3rd edn. The Williams \& Wilkins Company, Baltimore, pp 173-331

Bawa P, Chalmers GS, Stewart H, Eisen AA (2002) Responses of ankle extensor and flexor motoneurons to transcranial magnetic stimulation. J Neurophysiol 88:124-132 
Benecke R, Rothwell JPR, Dick BL, Marsden CD (1987) Disturbance of sequential movements in patients with Parkinson's disease. Brain 110:36-79

Brain L, Walton JN (1969) The Parkinson syndrome, in brain's diseases of the nervous system, 7th edn. Oxfore University Press, London, pp 522-534

Brodal A (1981) Neurological anatomy in relation to clinical medicine, 3rd edn. Oxford Univ Press, New York, p 257

Brown RG, Marsden CD (1988) Internal versus external cues and the control of attention in Parkinson's disease. Brain 111:323-345

Burleigh-Jacob A, Horak FB, Nutt SG, Obeso JA (1997) Step initiation in Parkinson's disease. Mov Disord 12:206-215

Carlsöö S (1966) The initiation of walking. Acta Anat 65:1-9

Carpinella I, Crenna P, Calabrese E, Rabuffetti M, Mazzoleni P, Nemni R (2007) Locomotor function in the early stage of Parkinson's disease. IEEE Trans Neural Syst Rehabil Eng 15:543-551

Chee R, Murphy A, Danoudis M, Georgiou-Karistianis N, Iansek R (2009) Gait freezing in Parkinson's disease and the stride length sequence effect interaction. Brain 132:2151-2160

Cioni M, Richards CL, Malouin F, Bedard PJ, Lemineux R (1997) Characteristics of the electromyographic patterns of lower limb muscles during gait in patients with Parkinosn's disease when off and on L-DOPA treatment. Ital J Neurol Sci 18:195-208

Crenna P, Carpinella I, Rabuffetti M, Rizzone M, Lopiano L, Lanotte M, Ferrarin M (2006) Impact of subthalamic nucleus stimulation on the initiation of gait in Parkinson's disease. Exp Brain Res 172:519-532

Crutcher MD, Alexander GE (1990) Movement-related neural activity selectively coding either direction or muscle pattern in three motor areas of monkey. J Neurophysiol 64:151-163

Crutcher MD, DeLong MR (1984) Single cell studies of the primate putamen. II. Relations to direction of movement and pattern of muscular activity. Exp Brain Res 53:244-258

Cummings JL, Steven JH (1992) Visuospatial abnormalities in Parkinson's disease. In: Huber SJ, Cummings JL (eds) Parkinson's disease neurobehavioral aspects. Oxford University Press, New York, pp 57-73

DeLong MR, Wichman T (2007) Circuits and circuit disorders of the basal ganglia. Arch Neurol 64:20-24

Dietz V, Colombo G, Jensen L, Baumgartner L (1995) Locomotor capacity of spinal cord in paraplegic patients. Ann Neurol 37:574-582

Elble RJ (1997) Changes in gait with normal aging. In: Masdeu JC, Sudarsky L, Wolfson L (eds) Gait disorders of aging. Falls and therapeutic strategies. Lippincott-Raven, New York, pp 93-105

Evarts EV, Teravainen H, Calone DB (1981) Reaction time in Parkinson's disease. Brain 104:167-186

Folstein MF, Folstein SE, McHugh PR (1975) "Mini-mental state". A practical method for grading the cognitive state of patients for the clinician. J Psychiatr Res 12:189-198

Gantchev N, Viallet F, Aurenty R, Massion J (1996) Impairment of posture-kinetic co-ordination during initiation of forward oriented stepping movement in parkinsonian patients. EEG Clin Neurophysiol 101:110-120

Georgopoulos AP, DeLong MR, Crutcher MD (1983) Relation between parameters of step-tracking movements and single cell discharge in the globus pallidus and subthalamic nucleus of the behaving monkey. J Neurosci 3:1586-1598

Halliday SE, Winter DA, Frank JS, Patla AE, Prince F (1998) The initiation of gait in young, elderly, and Parkinson's disease subject. Gait Posture 8:8-14

Harrington DL, Haaland KY (1991) Sequencing in Parkinson's disease: abnormalities in programming and controlling movement. Brain 114:99-115

Hikosaka O, Wurtz RH (1985) Modification of saccadic eye movements by GABA-related substances. I. Effect of muscimol and bicuculline in monkey superior colliculus. J Neurophysiol 53:266-291

Hikosaka O, Sakamoto M, Usui S (1989a) Functional properties of monkey caudate neurons. I. Activities related to saccadic eye movements. J Neurophysiol 61:780-798

Hikosaka O, Sakamoto M, Usui S (1989b) Functional properties of monkey caudate neurons. II. Visual and auditory properties. J Neurophysiol 61:799-813

Hoehn MM, Yahr MD (1967) Parkinsonism onset, progression, and mortality. Neurology 17:427-442

Hsu YT, Lai HY, Chang YC, Chiou SM, Lu MK, Lin YC, Liu YL, Chen CC, Huang HC, Chien TF, Lin SZ, Chen YY, Tsai CH (2012) The role of the sub-thalamic nucleus in the preparation of volitional movement termination in Parkinson's disease. Exp Neurol 233:253-263

Hughes AJ, Daniels SE, Kilford L, Lees AJ (1992) Accuracy of clinical diagnosis of idiopathic Parkinson's disease: clinico-pathological study of 100 cases. J Neurol Neurosurg Psychiatr 55:181-184

Jahn K, Deutschlander A, Stephan A, Kalla T, Huefner K, Wagner J, Strupp M, Brandt T (2008a) Supraspinal locomotor control in quadrupeds and humans. Prog Brain Res 17e:353-362

Jahn K, Deutschlander A, Stephan T, Kalla R, Wiesmann M, STrupp M, Brant T (2008b) Imaging human supraspinal locomotor centers in brainstem and cerebellum. Neuroimage 39:786-792

Kiriyama K, Warabi T, Kato M, Yoshida T, Kobayashi N (2004) Progression of human body sway during successive walking studied by recording sole-floor reaction forces. Neurosci Lett 359:130-132

Leblois A, Boraud T, Meissner W, Bergman H, Hnsel D (2006) Competition between feedback loops underlines normal and pathological dynamics in the basal ganglia. J Neurosci 26:3567-3583

Low KA, Miller J, Vierck E (2002) Response slowing in Parkinson's disease: a psychophysiological analysis of premotor and motor processes. Brain 125:1980-1994

Maertens de Noordhout A, Rapisarda G, Bogacz D, Gerard P, Pasqua VD, Pennisi G, Delwaide PJ (1999) Corticomotoneuronal synaptic connections in normal man: an electrophysiological study. Brain 122:1327-1340

Mink JW (1996) The basal ganglia: focused selection and inhibition of competing motor programs. Prog Neurobiol 50:381-425 (review)

Minkowski H (1908) Raum und Zeit. Jahresbericht der Deutschen Mathematiker Vereinigung 18:75-88

Naismith SL, Shine JM, Lewis SJ (2010) The specific contribution of set-shifting to freezing of gait in Parkinson's disease. Mov Disord 25:1000-1004

Nambu A (2008) Seven problems on the basal ganglia. Curr Opin Neurobiol 18:595-604 (review)

Nambu A, Kaneda K, Tokuno H, Takada M (2002a) Organization of corticostriatal motor inputs in monkey putamen. J Neurophysiol 88:1830-1842

Nambu A, Tokuno H, Takada M (2002b) Functional significance of the cortico-subthalamo-pallidal 'hyperdirect' pathway. Neurosci Res 43:111-117

Nantel J, McDonald JC, Tan S, Bronte-Stewart H (2012) Deficits in visuospatial processing contribute to quantitative measures of freezing of gait in Parkinson's disease. Neuroscience 221:151-156

Rodriguez-Oroz MC, Rodriguez M, Guridi J, Mewes K, Chockkman V, Vitek J, DeLong MR, Obeso JA (2001) The subthalamic nucleus in Parkinson's disease: somatotopic organization and physiological characteristics. Brain 124:1777-1790

Sard RC (1970) Relativistic mechanics. Q.A. Bejamin, Inc., New York

Takakusa K (2013) Neurophysiology of gait: from the spinal cord to the frontal lobe. Mov Disord 28:1483-1491 (review)

Warabi T, Kato M (2009) A new method to measure sole-floor reaction forces from anatomically discrete points of the sole during human locomotion. Recent Res Dev Neurosci 3:1-23 
Warabi T, Noda H, Yanagisawa N, Tashiro K, Shindo R (1986) Changes in sensory-motor function associated with the degree of bradykinesia of Parkinson's disease. Brain 109:1209-1224

Warabi T, Yanagisawa N, Shindo R (1988) Changes in strategy of aiming tasks in the Parkinson's disease. Brain 111:497-505

Warabi T, Kato M, Yoshida T, Kobayashi N (2004) Analysis of human locomotion by recording sole-floor reaction forces from anatomically discrete points. Neurosci Res 50:419-426

Warabi T, Fukushima K, Olley PM, Chiba S, Yanagisawa N (2011) Difficulty in terminating the preceding movement/posture explains the impaired initiation of new movements in Parkinson's disease. Neurosci Lett 496:84-89

Wichman T, Bergmann H, DeLong MR (1994) The primate subthalamic nucleus. I. Functional properties in intact animals. J Neurophysiol 72:496-506

Wing AM (1988) A comparison of the rate of pinch grip force increases and decreases in parkinsonian bradykinesia. Neuropsychologia 26:479-482 